\title{
Predicting Functional Outcome One Year After Traumatic Brain Injury With CT and MRI Findings
}

\author{
Tone Jerstad $^{\mathrm{a}}$, Cecilie Roe ${ }^{\mathrm{b}, \mathrm{c}}$, Paal Ronning ${ }^{\mathrm{d}}$, Solrun Sigurdardottir ${ }^{\mathrm{e}}$, \\ Per Nakstad ${ }^{\mathrm{a}}$, Nada Andelic ${ }^{\mathrm{b}, \mathrm{f}}$
}

\begin{abstract}
Background: The aim of this study was to evaluate the relative and combined impact of computed tomography (CT) and Magnetic resonance imaging (MRI) on functional outcome one year after traumatic brain injury (TBI).

Methods: This study was a prospective, population-based study of 87 patients with a Glasgow Coma Scale (GCS) score $\leq 12$ who were injured in 2005 - 2007 and hospitalized at Trauma Referral Centre in Eastern Norway. CT performed within $24 \mathrm{~h}$ post-injury was classified by Marshall classification scale. MRI performed one year post-injury was classified based on the presence or absence of diffuse axonal injuries (DAIs). The Glasgow Outcome Scale Extended (GOSE) was used as an outcome measure at the one-year follow-up. The predictions models were adjusted for clinical variables known to affect functional outcome.
\end{abstract}

Results: Using CT, small lesions (Marshall group 2) were observed in $37(42 \%)$ patients. Signs of increased intracranial pressure (Marshall groups 3 - 4) were present in $33(38 \%)$ patients. Using MRI, DAI lesions were found in $70 \%$ of patients. In the linear regression analysis that explored relative impact of CT, CT was a significant predictor of GOSE $(\mathrm{p}<0.001)$. In the model exploring combined impact of CT and MRI, MRI accounted for a larger proportion of variance in GOSE and appears as stronger predictor $(p<0.001)$

Manuscript accepted for publication August 17, 2012

${ }^{a}$ Department of Neuroradiology, Oslo University Hospital, Oslo, Norway

${ }^{\mathrm{b}}$ Department of Physical Medicine and Rehabilitation, Oslo University Hospital, Oslo, Norway

${ }^{c}$ Faculty of Medicine, University of Oslo, Oslo, Norway

${ }^{\mathrm{d} D e p a r t m e n t}$ of Neurosurgery, Oslo University Hospital, Ulleval, Oslo,

Norway

'Sunnaas Rehabilitation Hospital, Nesoddtangen, Norway

${ }_{\mathrm{f}}^{\mathrm{f}}$ Corresponding author: Nada Andelic, Department of physical medicine and rehabilitation, Oslo University Hospital, PB 4950 Oslo, Norway.

Email: nandelic@online.no

doi: http://dx.doi.org/10.4021/jnr133w than $\mathrm{CT}(\mathrm{p}=0.08)$.

Conclusions: The relative impact of CT findings of intracranial lesions in the acute settings and one-year MRI findings of DAI on functional outcome underscored the importance of using neuroimaging techniques when predicting functional outcomes after TBI. The better predictive value of MRI suggest that the detailed information about pathological brain lesions shown on late MR may help clinicians to administer more appropriate rehabilitation treatments to patients who are predicted to have a worse outcome at the one-year follow-up.

Keywords: Traumatic brain injury; CT; MRI; Functional outcome

\section{Introduction}

The neuropathology of traumatic brain injury (TBI) is complex, consisting of both various types and degrees of brain damage and structural lesions. Several neuroimaging classifications are used to classify structural brain lesions within TBI research, and most classifications are based on findings from acute computed tomography $(\mathrm{CT})[1,2]$. CT characterises injuries in a relatively broad set of categories and has significant prognostic value with regard to clinical outcome [3]. CT remains the modality of choice for the initial assessment in the detection of neurosurgical emergencies, e.g., fractures and acute hematomas $[4,5]$. The sensitivity of CT for detecting brain abnormalities in acute head trauma patients is between 63 and $73 \%$ [6]. However, it shows poor sensitivity in detecting abnormalities associated with milder brain injuries [7]. The presence, type and volume of intracranial mass lesions are associated with unfavourable outcomes after TBI $[1,8]$.

Magnetic resonance imaging (MRI) plays an important role in the diagnostics of post-traumatic intracranial lesions in the later phases of TBI [8-10]. MRI has the highest predictive power for evaluating sub-acute and chronic TBI and detecting axonal injuries [11]. Diffuse traumatic axonal injury (DAI) indicates extensive injury to white matter and occurs in approximately half of all severe TBI cases [12]. 
However, even the use of MRI will probably underestimate the incidence of DAIs. Newer imaging techniques, such as diffusion weighted imaging (DWI) and diffusion tensor imaging (DTI) have shown a potential to improve the detection of white matter injury in both acute and chronic DAI [13]. These methods help clinicians to explain impairments after TBI, guide clinical management and predict long-term outcomes $[14,15]$.

While publications regarding advances in MRI have increased, a relatively limited number of papers have evaluated the relationship between acute and chronic TBI-related brain lesions and long-term functional outcome after TBI. Some studies show poor correlation between MRI and CT [16], while other studies support the relationship between DAI and the depths of lesions detected by MRI in cases of unfavourable TBI outcome $[14,17]$. Few studies have studied the value of MRI in addition to established $\mathrm{CT}$ findings, taking other prognostic factors such as age and clinical aspects of injury severity into account $[18,19]$. From a rehabilitation perspective, such evaluation will aid the development and planning of appropriate rehabilitation strategies for patients with more severe functional deficits [3].

The aim of this study was to evaluate the relative and combined predictive values of CT and MRI on functional outcome one year after a TBI. Based on previous research, we expected that MRI would have a better predictive value than CT and that less severe brain lesions would equate to better functional outcome.

\section{Materials and Methods}

\section{Study design and participants}

This prospective cohort study is part of a larger TBI research project comprising patients with acute TBI admitted to Oslo University Hospital during a two year period from May 2005 to $2007[20,21]$.

The inclusion criteria were age (16 - 55 years); admittance to hospital with ICD-10 diagnoses of intracranial injuries (S06.0 - S06.9) within 24 hours post-injury; moderateto-severe TBI as assessed by Glasgow Coma Scale (GCS) score (GCS score $\leq 12$ ) before intubation [22]; CT of the brain performed within $24 \mathrm{~h}$ post-injury and MRI of the brain performed one year post-injury. Exclusion criteria were serious co-morbidities that interfered with the assessment of TBI-related disabilities such as previous neurological disorders, associated spinal cord injuries, previously diagnosed severe psychiatric or substance abuse disorders.

Of the 147 patients who fulfilled the inclusion criteria, 27 patients declined to participate, 21 patients died during acute care, two patients died during post-acute care, four patients dropped out of the study before the one-year followup and six patients did not conduct MRI scans because of claustrophobia or logistical difficulties. The remaining sample consisted of 87 patients from whom one year post-injury data were collected.

Independent variables: Demographic information was collected for the following items: age (continuous), gender (male vs. female), education ( $\leq 12$ years vs. $>12$ years), marital status (married/living together vs. living alone). The following injury characteristics were also obtained: cause of injury (traffic accidents vs. others), substance use at the time of injury (no vs. yes), injury severity: GCS score (continuous), Injury Severity Score (ISS) (continuous), dilated pupils (no vs. yes), hypoxia (oxygen saturation $\leq 90 \%$ ), hypotension (mean arterial pressure $\leq 60 \mathrm{mmHg}$ ), intracranial pressure measurement (ICP) (no vs. yes), length of acute hospital stay (days) and CT (Marshall classification). CT findings of epidural hematoma (EDH), subdural hematoma (SDH), traumatic subarachnoid haemorrhage (tSAH), intraventricular haemorrhage (IVH) (all no vs. yes), and MRI findings of DAI (DAI stages) and findings of cortical, and sub-cortical lesions and dilated periventricular space (all no vs. yes) were also recorded.

The ISS is an anatomical scoring system that provides an overall score for patients with multiple injuries [23]. The injuries within each body region are assigned to a 6 point ordinal Abbreviated Injury Scale (AIS) [24]. The ISS score is calculated as the sum of the squares of the highest AIS score in three different body regions, and ranges from 1 to 75 . An ISS of $\geq 15$ is universally accepted as the definition of a major trauma patient. The AIS and ISS scores were determined from the hospital trauma register [25].

CT head was performed shortly after acute admission, and a second CT was obtained within 6 to $24 \mathrm{~h}$ post-injury. They were taken with slice thickness of 5 to $10 \mathrm{~mm}$ in the axial plane. Findings from the first and second CT were categorised according to the diagnostic categories of anatomical abnormalities as classified by Marshall et al. [2]. The original Marshall classification ranges from 1 to 6 , with separate categories for any lesion that is surgically evacuated and non-evacuated mass lesions. The Marshall scores deduced from the "worst" CT scans were used in the final analyses. Patients scheduled for surgery were scored as evacuated mass lesions $[1,26]$.

MRI was performed with a $1.5 \mathrm{~T}$ (Magnetom Sonata Siemens, Erlangen, Germany) one year after TBI. The following imaging methods were used: T1-weighted spin-echo (SE), T2-weighted turbo SE, T2-weighted gradient echo (GRE), fluid-attenuated inversion recovery (FLAIR), inversion recovery (IR) T1 and diffusion-weighted imaging (DWI). Sagittal, transverse and coronal (multiplanar) images were obtaining with slice thicknesses varying from 5 to 7 $\mathrm{mm}$. Scans were interpreted and classified into $1-3$ stages of DAI. DAI stage 1 lesions confined to the lobar white matter are characterised by microscopic lesions localised at the level of the union of the grey and white matter and spare 
Table 1. Pre-analysis of Patients Characteristics (Independent Variables) in Relation to GOSE (Dependent Variable) One-year Post-injury $(n=87)$

\begin{tabular}{|c|c|c|c|}
\hline Independent variables & n (\%) & GOSE mean (SD) & P-value* \\
\hline Gender: $\mathrm{M} / \mathrm{F}$ & $65(75) / 32(25)$ & $5.7(2.0) / 5.9(2.3)$ & 0.12 \\
\hline Age at onset (years), mean (range) & $31(16-55)$ & & 0.27 \\
\hline \multicolumn{4}{|l|}{ Marital status (pre-injury) } \\
\hline Living with a spouse/ Living alone & $53(61) / 34(39)$ & $5.8(2.1) / 5.7(2.3)$ & 0.51 \\
\hline \multicolumn{4}{|l|}{ Education (pre-injury) } \\
\hline$\leq 12$ years $/>12$ years & $48(52) / 39(45)$ & $5.2(2.39 / 6.7(1.2)$ & 0.22 \\
\hline \multicolumn{4}{|l|}{ Cause of injury } \\
\hline Transport accidents/Others & $52(60) / 35(40)$ & $5.5(2.2) / 6.0(2.2)$ & 0.14 \\
\hline Substance use at injury time (no/yes) & $42(48) / 45(52)$ & $5.8(1.8) / 6.0(1.8)$ & 0.85 \\
\hline \multicolumn{4}{|l|}{ Severity of injury } \\
\hline GCS, mean (SD) & $7.1(3.3)$ & & $<0.001$ \\
\hline ISS, mean (SD) & $31.8(12.7)$ & & 0.001 \\
\hline Dilated pupils (no/yes) & $28(32) / 59(68)$ & $6.4(1.0) / 5.5(1.4)$ & 0.001 \\
\hline Hypoxemia (oxygen saturation $\leq 90 \%$ ), (no/yes) & $18(21) / 69(79)$ & $6.3(1.2) / 5.1(1.4)$ & $<0.001$ \\
\hline $\begin{array}{l}\text { Hypotension (mean arterial pressure } \leq 60 \mathrm{mmHg} \text { ), (no/ } \\
\text { yes) }\end{array}$ & $12(14) / 75(86)$ & $6.2(1.1) / 5.2(1.8)$ & 0.01 \\
\hline Intracranial pressure monitoring (ICP), (no/yes) & $51(59) / 36 / 41)$ & $6.7(0.8) / 5.6(1.4)$ & $<0.001$ \\
\hline CT head (Marshall score), mean (range) & $2.6(0-6)$ & & $<0.001$ \\
\hline Presence of $\mathrm{tSAH}$, (no/yes) & $57(65) / 30(35)$ & $6.0(1.8) / 4.9(2.3)$ & 0.03 \\
\hline Presence of EDH, (no/yes) & $18(21) / 69(79)$ & $5.1(2.3) / 5.8(2.1)$ & 0.25 \\
\hline Presence of SDH, (no/yes) & $44(50) / 43(50)$ & $5.7(2.1) / 4.8(2.3)$ & 0.02 \\
\hline Presence of IVH, (no/yes) & $29(34) / 58(66)$ & $5.7(2.2) / 4.4(2.2)$ & $<0.001$ \\
\hline Length of acute hospital stay, days median (IQR) & $23(30)$ & & $<0.001$ \\
\hline MRI findings one year post-injury, DAI mean (range) & $1.2(0-3)$ & & $<0.001$ \\
\hline Dilated perivascular space (no/yes) & $58(67) / 29(33)$ & $6.1(1.4) / 6.0(1.0)$ & 0.10 \\
\hline Cortical contusions (no/yes) & $30(35) / 57(65)$ & $6.2(1.3) / 6.0(1.3)$ & 0.57 \\
\hline Sub-cortical lesions (no/yes) & $68(78) / 19(22)$ & $6.3(1.0) / 4.9(1.6)$ & $<0.001$ \\
\hline
\end{tabular}

P-values* reported are from the simple regression analysis

the overlying cortex. At the more severe DAI stage 2, the corpus callosum is also involved. In severe DAI (stage 3), there are additional focal lesions in the dorsolateral quadrant of the rostral brainstem [27]. "Gliding" contusions caused by displacement of the grey matter of the cerebral cortex during acceleration of the head are often associated with DAI. The haemorrhages, which involve the deeper layer of the cortex and the convolutional white matter, are a type of shearing injury that occurs at the moment of impact [27]. Subcortical grey-matter lesions also result from shearing injury and are often seen in conjunction with the classical triad of DAI. The presence of cortical contusions, subcortical lesions and dilated perivascular spaces were therefore noted in this study.

The dependent variable in this study was the Glasgow Outcome Scale Extended (GOSE), which was assessed one year post-injury [28]. The GOSE divided patients into the following outcome categories: 1 = dead, 2 = vegetative state, $3=$ lower severe disability and complete dependence on others, $4=$ upper severe disability and some dependence on others, $5=$ lower moderate disability and working at a lower 


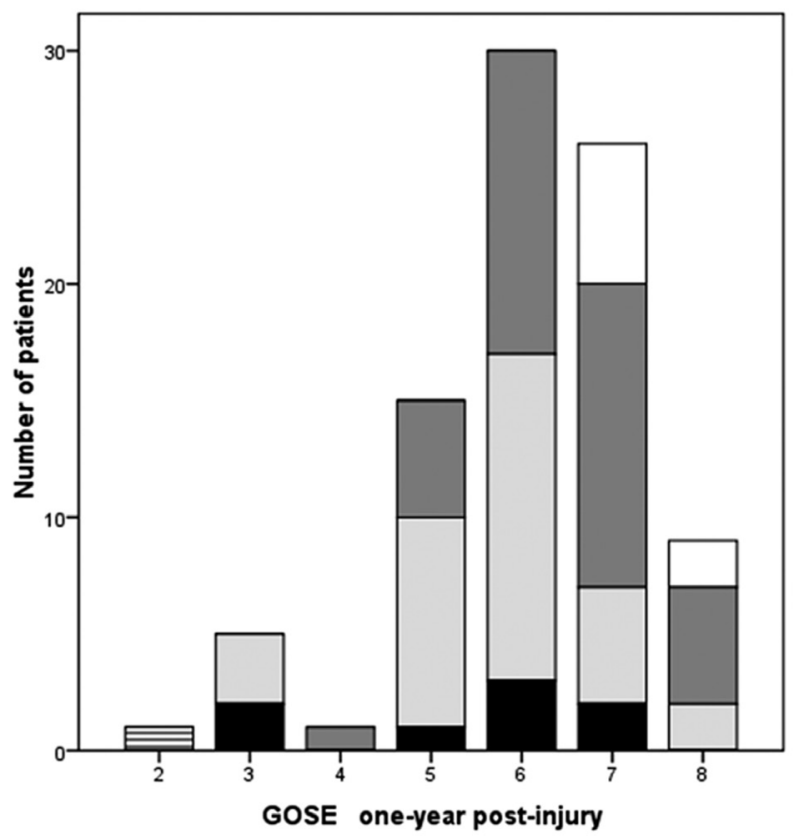

CT findings

$\square$ Marshal score 1

$\square$ Marshal score 2

$\square$ Marshal score 3

冒Marshal score 4

Marshal score 5

Figure 1. The distribution of CT findings as measured by Marshall scores across GOSE levels one year post-injury.

level of performance, $6=$ upper moderate disability and returning to previous work with some adjustments, $7=$ lower good recovery with minor physical or mental deficits and 8 $=$ upper good recovery.

\section{Procedure}

Demographic variables and injury-related characteristics including CT were collected during the acute hospital admissions. MRI was performed at one-year follow-up. A single neuroradiologist (TJ) reviewed the first and second head CT and classified findings according to the procedures in Marshall et al. (1992) and MRI according to the DAIs. Patients were interviewed and examined (NA) either in the outpatient clinic or in the rehabilitation hospital to determine GOSE outcome.

This study was approved by the Regional Committee for Medical Research Ethics and the Norwegian Data Inspectorate. Written informed consent was obtained from all participating patients.

\section{Statistical analysis}

Statistical analyses were performed using SPSS for Windows, version 16 (SPSS Inc., Chicago, IL). All statistical tests were two-sided, and a significance level of 0.01 was used because of the number of tests performed. Parametric statistics (t-tests) were chosen because the variables of importance (CT scan findings, MRI findings of DAI and GOSE) were normally distributed. Independent variables depicting patient characteristics are shown in Table 1. Univariate analyses including simple regression analysis were conducted to examine the relationship between the independent variables and GOSE outcome. Two multiple regression models using stepwise method were built using CT findings in the first model and MRI findings in the second model as predictors. Our third multiple regression model includes both CT and MRI as predictors. All models were adjusted for covariates that revealed a significance level of 0.01 in the simple regression analyses (see Table 1). The results are presented as an adjusted $\mathrm{R}^{2}$, B coefficients and standardised Beta $(\beta)$ values. The expected direction of the standardised $(\beta)$ is theorised to be negative for the CT and MRI findings, ISS and length of hospital stay, indicating that less severe injury equates to better functioning. Before conducting the multiple regression analysis, possible multicollinearities of the independent variables were examined using the variance inflation factor (VIF). The distribution of the residuals was examined for normality, and influential data points were examined using Cook's distance. The length of stay was log-transformed when performing linear regression analyses because this variable was skewed. None of the variables showed correlations among each other at $r>0.7$.

\section{Results}

Table 1 shows the demographic and clinical characteristics of the patients. The mean age at onset was 31 years, with $75 \%$ being male. Sixty per cent of patients were injured in 


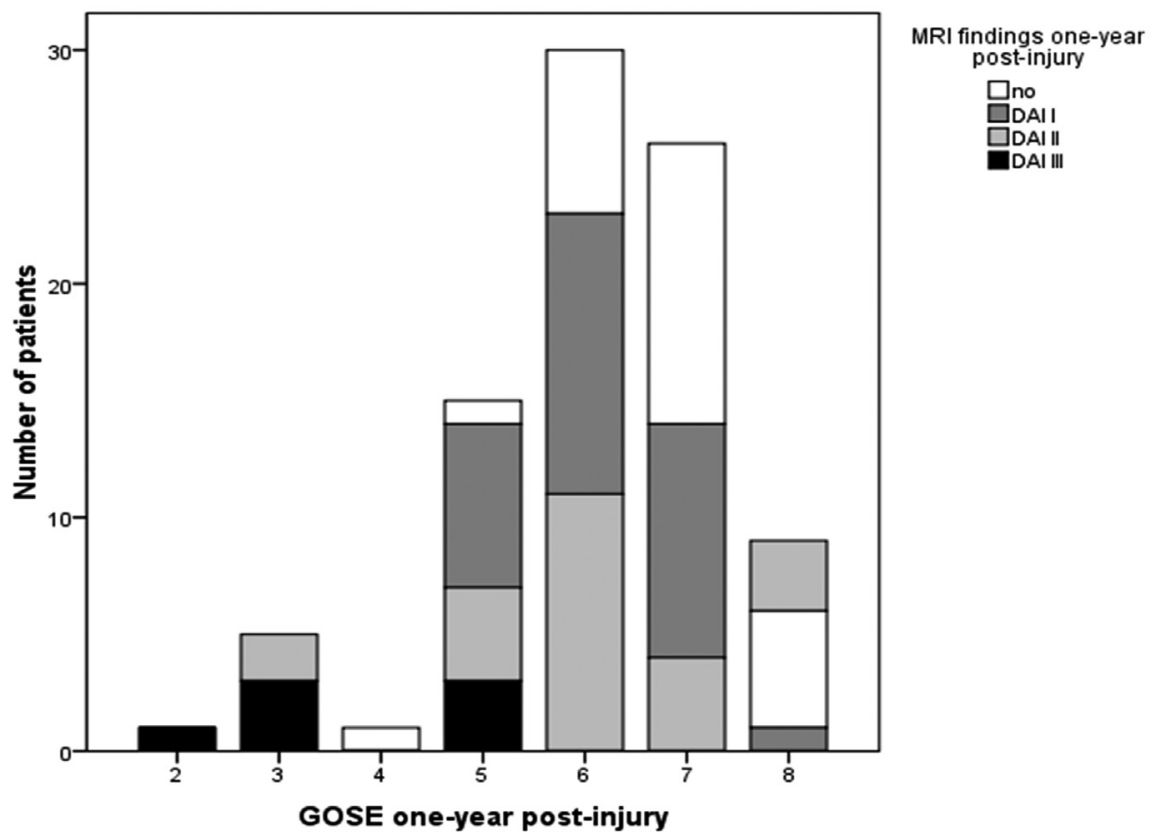

Figure 2. The distribution of MRI findings across GOSE levels one year post-injury.

traffic accidents. Three-quarters of patients had GCS within the range of severe TBI (GCS $3-8)$, and the majority of patients $(90 \%)$ were determined as being major trauma patients according to the ISS.

\section{CT findings}

Small lesions (Marshall group 2) were observed in 37 (42\%) patients. Signs of raised intracranial pressure (Marshall groups 3 - 4) were present in $33(38 \%)$ patients. Mass lesions (Marshall group 5) were excised from eight patients.
There was a high frequency of tSAH in this study $(65 \%)$. Cortical contusions were found in $56 \%$ of the patients, and sub-cortical lesions were found in $12 \%$. Indirect evidence of brain steam compression was found in half of the patients by evaluating the compression of the perimesencephalic cisterns and midline shift. A dorsolateral brain steam lesion was found in 3 patients. CT scans shown DAIs in $34 \%$ of the patients. Approximately $10 \%$ of patients showed increase of CT findings from the first to second CT performed within the first 6 - 24 h post-trauma. Eight patients had no visible intracranial pathology on the CT. Four of these patients showed

Table 2. Relationship Between CT, MRI and GOSE. Multivariate Stepwise Regression Model

\begin{tabular}{llll}
\hline \multicolumn{1}{c}{ Variables } & $\begin{array}{l}\text { B Coefficient } \\
\mathbf{9 5 \%} \mathbf{~ C I})\end{array}$ & $\beta$ & p-value \\
\hline Constant & 8.71 & $<0.001^{*}$ \\
CT & $-0.20(-0.42$ to 0.03$)$ & -0.16 & 0.08 \\
MRI & $-0.42(-0.66$ to -0.18$)$ & -0.31 & $0.001^{*}$ \\
Length of acute hospital stay & $-1.26(-1.80$ to -0.72$)$ & -0.42 & $<0.001^{*}$ \\
\hline
\end{tabular}

$\mathrm{R}^{2}$ for the model 0.46 ; Adjusted $\mathrm{R}^{2} 0.45$ 


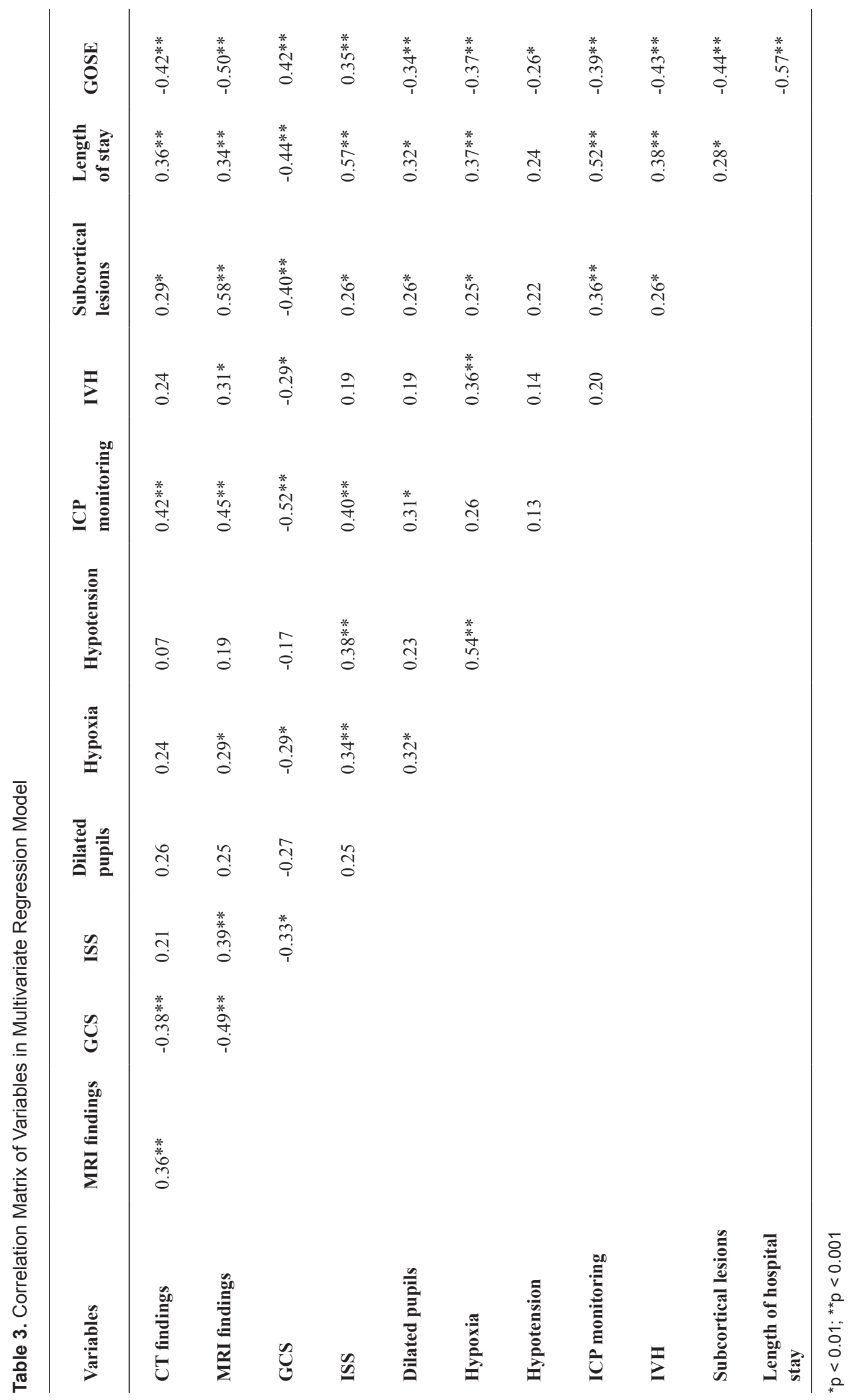


DAIs on MRI performed one year post-injury (three patients had stage 1 DAIs and one patient had stage 2 DAIs). A high frequency of DAI lesions on MRI was found in patients with IVH on CT $(86 \%)$ and tSAH $(72 \%)$.

\section{MRI findings}

MRI showed DAIs in $70 \%$ of patients (48\% in patients with GCS 9 - 12 and 83\% in patients with GCS 3 - 8). The most frequent DAI stage was stage 1 in 30 patients (48\%), followed by stage 2 in 24 patients (40\%) and stage 3 in seven patients $(12 \%)$. Haemorrhagic DAIs were found in $85 \%$ of patients. Dilated perivascular spaces were seen in $47 \%$ of DAI patients. Combined DAI and cortical lesions were observed in $42 \%$ of patients, while $12 \%$ of patients had combined DAIs and sub-cortical lesions. Of 25 patients in the "no DAIs group", 50\% had cortical contusions on MRI. Patients with DAIs were more frequently injured in transport accidents $(60 \%) ; 56 \%$ of these patients had stage 2 - 3 DAI lesions.

\section{Functional outcome one year post-injury}

The mean GOSE at the one year follow-up was 5.8 (SD 2.2). Eight per cent of patients suffered severe disability (GOSE 2 - 4), 52\% showed moderate disability (GOSE 5 - 6) and $40 \%$ showed good recovery (GOSE 7 - 8). The relationship between the patients' characteristics and GOSE is shown in Table 1.

The distribution of CT pathology across the eight GOSE levels is shown in Figure 1, and the distribution of MRI findings is presented in Figure 2. Of seven patients who were in the severe range of GOSE (level 2 - 4) one year post-injury, three patients had a Marshall score of $4-5$ on the CT scan showing most traumatic changes. Of 35 patients showing good recovery (GOSE 7 - 8), two patients had a Marshall score of 5, and seven patients a Marshall score of 3; the remaining 26 patients had no visible or small lesions.

The mean GOSE was 6.7 (SD 0.96) in patients without DAI lesions and 5.8 (SD 1.3) in patients with DAIs. Of all patients with DAIs, 31\% showed good recovery (GOSE 7 8 ) in contrast to $64 \%$ of patients without DAIs $(p=0.001)$. Of those patients with good recovery $(n=35), 21 \%$ patients had a stage 2 DAI, and $30 \%$ had a stage 1 DAI. Four of seven patients with severe disability (GOSE 2 - 4) had a stage 3 DAI on MRI, while two patients had a stage 2 DAI.

In the regression analysis that explored the impact of CT (Marshall score 1 - 5) on GOSE, CT was a significant predictor, with negative beta values $(\beta=-0.42, \mathrm{p}<0.001)$, meaning that less severe injury leads to better functional outcomes. CT accounted for $17 \%$ of the variance in the GOSE for the simple regression analysis. The multivariate linear regression model explained $40 \%$ of the variance in GOSE. In addition to $\mathrm{CT}(\beta=-0.22, p=0.01)$, two covariates used in the multivariate model, IVH and length of acute hospital stay, were also significant predictors, with negative $\beta$-value $(\beta=$ $-0.23, p=0.01$ and $\beta=-0.40, p<0.001$, respectively), meaning that less severe intracranial injury, without IVH and with a shorter stay in acute care, leads to better GOSE outcomes.

In the regression analysis exploring the effect of MRI (DAI stages 1 - 3) on GOSE, MRI was a significant predictor, with negative beta value $(\beta=-0.50 ; \mathrm{p}<0.001)$, indicating that less severe injury leads to better functional outcome. The MRI accounted for $25 \%$ of the variance in the GOSE in the simple regression analysis. The multivariate linear regression model explained $45 \%$ of the variance in the GOSE. In addition to MRI $(\beta=-0.32 ; p=0.001)$, length of stay was a significant predictor, with a negative direction of the $\beta$-value $(\beta=-0.40 ; \mathrm{p}<0.001)$.

In the third regression model exploring the combined impact of CT and MRI on the GOSE, MRI accounted for $25 \%$ of the variance in the GOSE, while CT explained $7 \%$. The final multivariate model accounted for $45 \%$ of the variance, resulting in two significant predictor variables: the length of acute hospital stay and MRI (Table 2).

The multivariate linear regression analyses that were performed to explore the impact of CT and MRI on GOSE in the subgroup with DAI showed similar predictors as the abovementioned model (data not shown).

The multicollinearity diagnostic indicated an acceptable degree of collinearity (see Table 3 for a correlation matrix of all variables) with a VIF of $<1.2$ and Cook's distance $<0.14$.

\section{Discussion}

\section{CT findings}

The dynamic nature of TBI can be characterised by taking a serial CT examinations. Marshall et al. [2] developed a more discrete classification of head injury, not only for prognostic purposes, but also as a tool to assist in the diagnosis and treatment of secondary injuries, which are avoidable and can occur at variable times after the initial injury.

Approximately $10 \%$ of the patients with initial Marshall 1 - 2 lesions developed new changes on the second CT scan performed within $24 \mathrm{~h}$ post-injury, which is in accordance with a study by Lagares et al. [14]. We did not evaluate all CT scans from the acute hospitalisation period, but previous studies have reported that up to $50 \%$ of patients may develop new lesions during the acute phase [29]. The increasing severity of brain injury is indicated on the CT by indirect evidence of brain stem compression, the size of the perimesencephalic cisterns and a midline shift [2]. In fifty-nine per cent of patients monitoring with an ICP during the acute hospitalisation was regarded necessary, and may indicate that the percentage of newly developed lesions in our study was underestimated. CT also seems to underestimate the sever- 
ity of cerebral injuries and has low sensitivity in visualising DAIs and especially non-haemorrhagic lesions [15].

In our study, comparison with the one year MRIs indicates that $\mathrm{CT}$ underestimated the presence of brain lesions in four of eight patients and DAIs in 36\% of the patients. Previous studies have reported that DAIs are detected on CT scans in $20-50 \%$ of cases [9]. Several studies, however, have indicated the importance of examining individual CT factors and not just categorising findings according to the Marshall Classification [30]. The percentage of tSAH in this study was high $(65 \%)$ but within the range of previous studies $(28-79 \%)$ $[1,14]$. Our results demonstrate the relationship between the presence of tSAH, cortical and subcortical lesions, IVH and DAI. IVH is an important predictor of functional outcome in the univariate analyses in this study, in accordance with previous studies $[1,14]$.

\section{MRI findings}

MRI is sensitive for the detection of primary intra axial lesions and white matter shear injuries. Primary classical triad of DAI findings mentioned earlier are especially noted here. Studies report that the incidence of DAIs on MRI is in the range of $65-72 \%[14,17]$. Our findings of $70 \%$ DAI lesions are in accordance with these studies and other findings showing that DAIs are more frequent in high-energy trauma events. Our results may be an underestimate because we did not perform MRI in the acute phase $[14,15,16]$. Oedema associated with non-haemorrhagic DAI or small haemorrhagic DAI lesions demonstrated on early MRI may have resolved and become undetectable at later repeated MRI scans performed weeks and months post-injury [31]. Axonal injury by itself can occur without concomitant significant vascular injury [32]. The propensity for a white matter shearing lesion to haemorrhage partially depends on the degree of vascularity of the injured area. When a shearing injury to a wellvascularised area is non-haemorrhagic, it is conceivable that only the perivascular space surrounding the perforated vessel is interrupted. A stronger shearing force will disrupt both the perivascular space and the vessel wall and result in a haemorrhagic lesion with surrounding oedema [27, 33]. The majority of DAI lesions in this study were haemorrhagic, even though we know from the pathological literature that most lesions are non-haemorrhagic (80\%) [34]. Diffusion-weighted images is very sensitive for detecting non-haemorrhagic lesions, but as in the case with ischaemia, its sensitivity is limited to the acute setting [35]. Chronic DAI lesions are either invisible or show decreased signal. Perivascular spaces have a tendency to dilate in healthy patients but can also be associated with diseases and trauma [36]. The visibility on T2-weighted gradient-echo imaging is time independent, which allows recognition of these lesions during the chronic stage of TBI [11].

Our standard image analysis protocols do not truly characterise the full extent or consequences of DAIs [37]. Although MRI is far more sensitive than CT for detecting shearing injuries, microscopically recognisable axonal damage may not be identified by either modality [37].

\section{Functional outcome one year post-injury}

Patients with less severe brain injury findings on CT showed better GOSE outcomes in accordance with previous studies $[1,38]$. The present study indicates that patients with MRI findings of stage 1-2 DAIs may have good functional outcome one year post-TBI, while patients with stage 3 DAIs had poorer GOSE outcome, which is in accordance with previous studies [14, 39]. However, our study showed that DAI lesions are more frequent in patients with an unfavourable outcome as evaluated by GOSE levels 2 - 5. Other studies have reported a correlation between functional outcome as assessed by GOS/GOSE and CT and MRI findings [14, 15, 17]. In contrast to these studies, another study using MRI two years post-injury showed a lack of correlation between the number and site of traumatic microbleeds and an overall long-term clinical outcome such as the GOSE [11].

A novel feature of this study is the use of multivariate regression to assess the contributions of acute $\mathrm{CT}$ and late MRI findings to functional outcome one year after a TBI. We performed an adjustment for clinical variables that are important for functional outcome after TBI, such as clinical evidence of acute injury severity. Despite the significant association of CT with GOSE outcome, the CT did not independently contribute to outcome when MRI was included in the regression model. MRI was a significant predictor and accounted for $25 \%$ of the variance in GOSE. A high incidence of DAIs in our MRI data may give extra power in the model. Length of hospital stay in acute care was a consistent significant predictor of functional outcome one year postinjury and agreed with other studies on functional outcome after TBI [26]. Unexpectedly, individual CT characteristics such as tSAH and IVH were not significant predictors in the multivariate analysis, possibly due to the limited number of patients included in this study. Age did not influence functional outcome, which contradicts other studies [40]. A likely explanation is the exclusion of older patients ( $>55$ years) from the current study.

Half of the variance in the multivariate models remained unexplained in this study and may be explained by the use of the GOSE as global functional outcome. Variables of significant importance such as cognitive and psychosocial consequences of TBI were not examined in this study. With better methods to image the functional capacity of the brain and further refine the neuroimaging classification, it is likely that the outcomes of patients will improve and that outcome prediction will be more accurate.

From a rehabilitation perspective, MRI is not readily available in the early phases of TBI, and early CT scans are 
often the only scans performed from which physiatrists make predictions during inpatient rehabilitation. An important question would be whether the assessment of a late MRI has any additional value over an early $\mathrm{CT}$ in providing prognostic information for patients with moderate-to-severe TBI. In general, physiatrists are often consulted by patients or care providers one year post-injury due to persistent functional impairments and an inability to return to work. An MRI obtained in this period will have an additional value by disclosing DAI lesions that were not shown on CT or other subtle findings. This information may aid the rehabilitation team in setting appropriate goals for rehabilitation intervention and realistic outcome expectations [41].

Several limitations should be addressed in this study. The study has been conducted in an age-selected population (16 - 55 years). The use of a single neuroradiologist (TJ) to rate both the CT and MRI could introduce a systematic bias. However, the neuroradiologist was blinded for the outcome variable. Additionally, we did not monitor the progression in $\mathrm{CT}$ examinations performed after the first day of injury. We found a limited number of patients with stage 3 DAIs on MRI, possibly because we included only patients who survived one year follow-up. Therefore, we did not separate and categorise DAI severity in the regression analyses. Our results need to be validated using a larger sample of unselected TBI patients and a more detailed functional outcome measures.

\section{Conclusions}

The relative impact of early CT findings of intracranial lesions and late MRI findings of DAI on functional outcome supported results from previous studies and underscored the importance of using neuroimaging techniques when predicting functional outcomes after TBI. The better predictive value of MRI indicates that the detailed information about pathological brain lesions shown on late MRI may help clinicians to administer more appropriate rehabilitation treatments to patients who are predicted to have a worse outcome at the one-year follow-up.

\section{Acknowledgement}

The authors would like to thank the patients for their participation. Special thanks are given to Morten Hestnes for the extraction of trauma scores from Trauma Register, Oslo University Hospital, Ulleval. The Norwegian Health SouthEast Authority granted this study.

\section{Conflicts of Interest}

None.

\section{References}

1. Maas AI, Steyerberg EW, Butcher I, Dammers R, Lu J, Marmarou A, Mushkudiani NA, et al. Prognostic value of computerized tomography scan characteristics in traumatic brain injury: results from the IMPACT study. J Neurotrauma. 2007;24(2):303-314.

2. Marshall LF, Marshall SB, Klauber MR, Van Berkum Clark M, Eisenberg H, Jane JA, Luerssen TG, et al. The diagnosis of head injury requires a classification based on computed axial tomography. J Neurotrauma. 1992;9 Suppl 1:S287-292.

3. Haacke EM, Duhaime AC, Gean AD, Riedy G, Wintermark M, Mukherjee P, Brody DL, et al. Common data elements in radiologic imaging of traumatic brain injury. J Magn Reson Imaging. 2010;32(3):516-543.

4. Besenski N. Traumatic injuries: imaging of head injuries. Eur Radiol. 2002;12(6):1237-1252.

5. Lee TT, Aldana PR, Kirton OC, Green BA. Follow-up computerized tomography (CT) scans in moderate and severe head injuries: correlation with Glasgow Coma Scores (GCS), and complication rate. Acta Neurochir (Wien). 1997;139(11):1042-1047; discussion 10471048.

6. Metting Z, Rodiger LA, De Keyser J, van der Naalt J. Structural and functional neuroimaging in mild-to-moderate head injury. Lancet Neurol. 2007;6(8):699-710.

7. Bigler ED, Blatter DD, Johnson SC, Anderson CV, Russo AA, Gale SD, Ryser DK, et al. Traumatic brain injury, alcohol and quantitative neuroimaging: preliminary findings. Brain Inj. 1996;10(3):197-206.

8. Jacobs B, Beems T, van der Vliet TM, Diaz-Arrastia RR, Borm GF, Vos PE. Computed tomography and outcome in moderate and severe traumatic brain injury: hematoma volume and midline shift revisited. J Neurotrauma. 2011;28(2):203-215.

9. Gallagher CN, Hutchinson PJ, Pickard JD. Neuroimaging in trauma. Curr Opin Neurol. 2007;20(4):403-409.

10. Levine B, Kovacevic N, Nica EI, Cheung G, Gao F, Schwartz ML, Black SE. The Toronto traumatic brain injury study: injury severity and quantified MRI. Neurology. 2008;70(10):771-778.

11. Scheid R, Preul C, Gruber O, Wiggins C, von Cramon DY. Diffuse axonal injury associated with chronic traumatic brain injury: evidence from $\mathrm{T} 2 *$-weighted gradient-echo imaging at $3 \mathrm{~T}$. AJNR Am J Neuroradiol. 2003;24(6):1049-1056.

12. Jennett B, Adams JH, Murray LS, Graham DI. Neuropathology in vegetative and severely disabled patients after head injury. Neurology. 2001;56(4):486-490.

13. Le TH, Gean AD. Neuroimaging of traumatic brain injury. Mt Sinai J Med. 2009;76(2):145-162.

14. Lagares A, Ramos A, Perez-Nunez A, Ballenilla F, Alday R, Gomez PA, Kaen A, et al. The role of MR im- 
aging in assessing prognosis after severe and moderate head injury. Acta Neurochir (Wien). 2009;151(4):341356.

15. Marquez de la Plata C, Ardelean A, Koovakkattu D, Srinivasan P, Miller A, Phuong V, Harper C, et al. Magnetic resonance imaging of diffuse axonal injury: quantitative assessment of white matter lesion volume. J Neurotrauma. 2007;24(4):591-598.

16. Newberg AB, Alavi A. Neuroimaging in patients with head injury. Semin Nucl Med. 2003;33(2):136-147.

17. Skandsen T, Kvistad KA, Solheim O, Lydersen S, Strand IH, Vik A. Prognostic value of magnetic resonance imaging in moderate and severe head injury: a prospective study of early MRI findings and one-year outcome. J Neurotrauma. 2011;28(5):691-699.

18. Chelly H, Chaari A, Daoud E, Dammak H, Medhioub F, Mnif J, Hamida CB, et al. Diffuse axonal injury in patients with head injuries: an epidemiologic and prognosis study of 124 cases. J Trauma. 2011;71(4):838846.

19. Nelson DW, Nystrom H, MacCallum RM, Thornquist B, Lilja A, Bellander BM, Rudehill A, et al. Extended analysis of early computed tomography scans of traumatic brain injured patients and relations to outcome. J Neurotrauma. 2010;27(1):51-64.

20. Andelic N, Sigurdardottir S, Brunborg C, Roe C. Incidence of hospital-treated traumatic brain injury in the Oslo population. Neuroepidemiology. 2008;30(2):120128.

21. Sigurdardottir S, Andelic N, Roe C, Schanke AK. Cognitive recovery and predictors of functional outcome 1 year after traumatic brain injury. J Int Neuropsychol Soc. 2009; 15(5):740-750.

22. Teasdale G, Jennett B. Assessment of coma and impaired consciousness. A practical scale. Lancet. 1974;2(7872):81-84

23. Baker SP, O’Neill B, Haddon W, Jr., Long WB. The injury severity score: a method for describing patients with multiple injuries and evaluating emergency care. J Trauma. 1974;14(3):187-196.

24. Byrd DM, 3rd, Allen DO, Beamer RL, Besch HR, Jr., Bylund DB, Doull J, Fleming WW, et al. The dose-response model for dioxin. Risk Anal. 1998;18(1):1-2.

25. Skaga NO, Eken T, Steen PA. Assessing quality of care in a trauma referral center: benchmarking performance by TRISS-based statistics or by analysis of stratified ISS data? J Trauma. 2006;60(3):538-547.

26. Sandhaug M, Andelic N, Vatne A, Seiler S, Mygland A. Functional level during sub-acute rehabilitation after traumatic brain injury: course and predictors of outcome. Brain Inj. 2010;24(5):740-747.

27. Adams JH, Graham DI, Murray LS, Scott G. Diffuse axonal injury due to nonmissile head injury in humans: an analysis of 45 cases. Ann Neurol. 1982;12(6):557-563.
28. Wilson JT, Pettigrew LE, Teasdale GM. Structured interviews for the Glasgow Outcome Scale and the extended Glasgow Outcome Scale: guidelines for their use. J Neurotrauma. 1998;15(8):573-585.

29. Lobato RD, Alen JF, Perez-Nunez A, Alday R, Gomez PA, Pascual B, Lagares A, et al. [Value of serial CT scanning and intracranial pressure monitoring for detecting new intracranial mass effect in severe head injury patients showing lesions type I-II in the initial CT scan]. Neurocirugia (Astur). 2005;16(3):217-234.

30. Maas AI, Hukkelhoven CW, Marshall LF, Steyerberg EW. Prediction of outcome in traumatic brain injury with computed tomographic characteristics: a comparison between the computed tomographic classification and combinations of computed tomographic predictors. Neurosurgery. 2005;57(6):1173-1182; discussion 11731182.

31. Levine B, Fujiwara E, O'Connor C, Richard N, Kovacevic N, Mandic M, Restagno A, et al. In vivo characterization of traumatic brain injury neuropathology with structural and functional neuroimaging. J Neurotrauma. 2006;23(10):1396-1411.

32. Johnson VE, Stewart W, Smith DH. Axonal pathology in traumatic brain injury. Exp Neurol. 2012.

33. Gennarelli TA, Spielman GM, Langfitt TW, Gildenberg PL, Harrington T, Jane JA, Marshall LF, et al. Influence of the type of intracranial lesion on outcome from severe head injury. J Neurosurg. 1982;56(1):26-32.

34. Gentry LR, Godersky JC, Thompson B. MR imaging of head trauma: review of the distribution and radiopathologic features of traumatic lesions. AJR Am J Roentgenol. 1988;150(3):663-672.

35. Schaefer PW, Huisman TA, Sorensen AG, Gonzalez $\mathrm{RG}$, Schwamm LH. Diffusion-weighted MR imaging in closed head injury: high correlation with initial glasgow coma scale score and score on modified Rankin scale at discharge. Radiology. 2004;233(1):58-66.

36. Inglese M, Bomsztyk E, Gonen O, Mannon LJ, Grossman RI, Rusinek H. Dilated perivascular spaces: hallmarks of mild traumatic brain injury. AJNR Am J Neuroradiol. 2005;26(4):719-724.

37. Sidaros A, Engberg AW, Sidaros K, Liptrot MG, Herning $\mathrm{M}$, Petersen $\mathrm{P}$, Paulson OB, et al. Diffusion tensor imaging during recovery from severe traumatic brain injury and relation to clinical outcome: a longitudinal study. Brain. 2008;131(Pt 2):559-572.

38. Maas AI, Stocchetti N, Bullock R. Moderate and severe traumatic brain injury in adults. Lancet Neurol. 2008;7(8):728-741.

39. Pierallini A, Pantano P, Fantozzi LM, Bonamini M, Vichi R, Zylberman R, Pisarri F, et al. Correlation between MRI findings and long-term outcome in patients with severe brain trauma. Neuroradiology. 2000;42(12):860867. 
40. Flanagan SR, Hibbard MR, Gordon WA. The impact of age on traumatic brain injury. Phys Med Rehabil Clin N Am. 2005;16(1):163-177.

41. Englander J, Cifu DX, Wright JM, Black K. The asso- ciation of early computed tomography scan findings and ambulation, self-care, and supervision needs at rehabilitation discharge and at 1 year after traumatic brain injury. Arch Phys Med Rehabil. 2003;84(2):214-220. 